\title{
If food is our medicine and medicine is our food - what about chocolate
}

Volume 7 Issue 3 - 2017

\author{
Holly Fourchalk \\ Choices unlimited for Health \& Wellness Ltd, Canada
}

\section{Opinion}

As nutritionists, we are taught that the highest source of nutrient in any food or herb is in the seed. Chocolate is no different. The cocoa pod is a fruit full of juice and anywhere between 12-6800 seeds, depending on the species. Initially, all species were referred to as Theobroma cacao meaning (theo) God and (brosi) food or "food of the gods". These seeds are a hugely nutrient dense food. They contain over 1200 compounds and over 300 nutrients that we need. Not too bad. Unfortunately, Western society learned how to eliminate the nutrients through pasteurization. Then adds various toxic chemicals including sugars. When marketers claim that the chocolate is $60 \%$, $72 \%, 84 \%$ chocolate, etc., what they fail to explain is that the really healthy omega $3 \mathrm{~s}$ were extracted and usually sold to nutraceutical companies AND the process eliminated upwards of $80 \%$ of the antioxidants for which the dark chocolate is marketed for! Real $100 \%$ chocolate contains vitamins, minerals, all the amino acids (essential \& non-essential) fiber, omega 3 fatty acids, anti-oxidants (2x more than acai berry), bioflavonoids and much more. What do these nutrients do for you? Besides taste great?

\section{Fights weight}

a. Regulates the two important hormones in weight metabolism: grehlin \& leptin;

b. Opens fat cells so that they release stored toxins

c. Alters the genetic coding changing the fat cell into fatty acids used for energy.

\section{Fights cancer}

a. Turns on apoptosis"automatic cell death"

b. Changes the membrane so it receives oxygen instead of sugar

c. Blocks off arterial nutrients - starves the cells

d. Alkalizes the interior and exterior of the cell.

\section{Fights heart disorders}

a. Re-stabilizes oxidized cholesterols;

b. Induces vasodilation

c. Lowers blood pressure

d. Improves coronary vascular function

e. Decreases platelet adhesion - reduces arteriosclerosis plaque.

\section{Fights diabetes}

a. High levels of soluble \& non-soluble fibers slow down sugar absorption;

\author{
Correspondence: Holly Fourchalk, Choices unlimited for \\ Health \&Wellness Ltd, Canada, Tel 604 764-5203, \\ Email holly@choicesunlimited.ca
}

Received: February 10, 2017 | Published: May 30, 2017

b. Diabetics are always low in anti-oxidants and consequently high in damaging free radicals, $100 \%$ chocolate is a good source of a variety of anti-oxidants.

\section{Fights digestive issues, bowel issues, gastric} issues

a. Provides huge amounts of micro probiotics, fibers/prebiotics;

b. Protects the gut from acidity and alkalizes our gastro tract so that the good bacteria can thrive;

c. Eliminates inflammation that disrupts the environment for healthy bacteria;

d. Inhibits the growth of bad bacterial growth in the gut.

\section{Fights psychological issues}

a. Provides all the amino acids required to make serotonin, neuroephinephrine, dopamine and other neurotransmitters;

b. Provides endorphins and natural anti-depressants, ie., MAOIs

c. The brain is between $60-70 \%$ fats and $100 \%$ provides the healthy omega 3 /fatty acid fats.

The cocoa bean contains several useful minerals and vitamins

a. Magnesium, which is necessary for muscle relaxation, nerve conduction, energy production and bone and teeth development and overall health;

b. Copper-found in rich supply in dark cocoa-is involved in many of the chemical processes in the body;

c. High levels of potassium, which is vital for cardiovascular health.

Consequently, when we choose the right chocolate, it can be a huge benefit to our physical and psychological health. 


\section{Acknowledgments}

None.

\section{Conflicts of interest}

Author declares there are no conflicts of interest.

\section{Funding}

None. 